\title{
Neuralgia del trigémino provocada por un tumor del ángulo ponto-cerebeloso contralateral. Caso clínico
}

\author{
C. Chamadoira; A. Cerejo; F. Duarte y R. Vaz
}

Departamento de Neurocirugia. Hospital de São João. Porto. Portugal.

\section{Resumen}

La gran mayoría de neuralgias del trigémino son atribuidas a la compresión por estructuras vasculares, de la zona de entrada del nervio a nivel de la protuberancia. En torno al $1 \%$ de los casos de neuralgia del trigémino están asociados a tumores del ángulo pontocerebeloso ipsilaterales. Las neuralgias del trigémino provocadas por tumores contralaterales de la fosa posterior son muy raras. Los autores presentan el caso clínico de un paciente de 37 años, con una neuralgia del trigémino derecha y un voluminoso neurinoma del acústico izquierdo. El dolor remitió completamente después de la escisión del tumor.

PALABRAS CLAVE: Ángulo ponto-cerebeloso. Dolor contralateral. Neuralgia del trigémino. Neurinoma acústico.

Trigeminal neuralgia caused by contra lateral cerebellopontine angle tumor. A case report

\section{Summary}

In most cases, trigeminal neuralgia is due to compression of the trigeminal nerve in the zone of entrance at the pons by vascular structures. About $1 \%$ of cases, have trigeminal neuralgia associated with the presence of ipsilateral lesion of cerebellar-pontine angle. Rarely, trigeminal neuralgia may be due to contralateral posterior fossa tumors. We present a case of a 37-year- old patient who presented with right trigeminal neuralgia and harboured a left acoustic neuroma of significant dimension. Facial pain completely disappeared after tumor removal.

KEY WORDS: Acoustic neuroma. Cerebellopontine angle. Contra lateral pain. Trigeminal neuralgia.

Recibido: 27-07-09. Aceptado: 30-10-09

\section{Introducción}

La neuralgia del trigémino, también llamada "tic doloroso", es un fenómeno relativamente común. Su incidencia anual ha sido estimada en torno a 1/25.000 adultos ${ }^{13}$. El dolor facial está relacionado con la compresión del nervio trigémino en su zona de entrada en la protuberancia, por vasos, tumores o adherencias aracnoideas. En el año 1910 , Weisenburg ${ }^{12}$ describió por primera vez la relación entre neuralgia del trigémino y tumores del ángulo pontocerebeloso del mismo lado. Varios estudios clínicos indican que, en torno al $1 \%$ de los pacientes con neuralgia del trigémino, presentan un tumor ipsilateral del ángulo pontocerebeloso.

La neuralgia del trigémino asociada a tumores contralaterales es extremadamente infrecuente ${ }^{1,7,10}$. En el año 1987 Florensa y $\mathrm{col}^{4}{ }^{4}$ en una revisión de la literatura, identificaron 29 casos a los que estos autores añadieron 3 más.

Presentamos el caso clínico de un paciente con un neurinoma del acústico izquierdo, que presentaba una neuralgia del trigémino derecho. El dolor facial remitió completamente después de la escisión de la lesión.

\section{Caso clínico}

Paciente de sexo masculino, de 37 años de edad, sin antecedentes patológicos relevantes y con historia de dolor facial derecho con 2 meses de evolución. El dolor es descrito como paroxístico, lancinante y afectando a la $3^{\text {a }}$ división del nervio trigémino. La masticación así como ciertos movimientos de la musculatura facial, desencadenaban el dolor llegando a provocarle náuseas y vómitos. Fue inicialmente medicado con Carbamazepina y Gabapentina, sin alivio del dolor.

El examen neurológico mostraba una ligera hipoestesia en el territorio de la tercera rama del V par derecho y una hipoacusia sensitiva izquierda. Se solicita una RM cerebral que reveló una lesión de $4,5 \times 3,5 \mathrm{~cm}$ en la cisterna pontocerebelosa izquierda, que captaba contraste intensamente y cuyos contornos eran nítidos. Esta lesión condicionaba una marcada compresión y rotación del tronco cerebral hacia la 


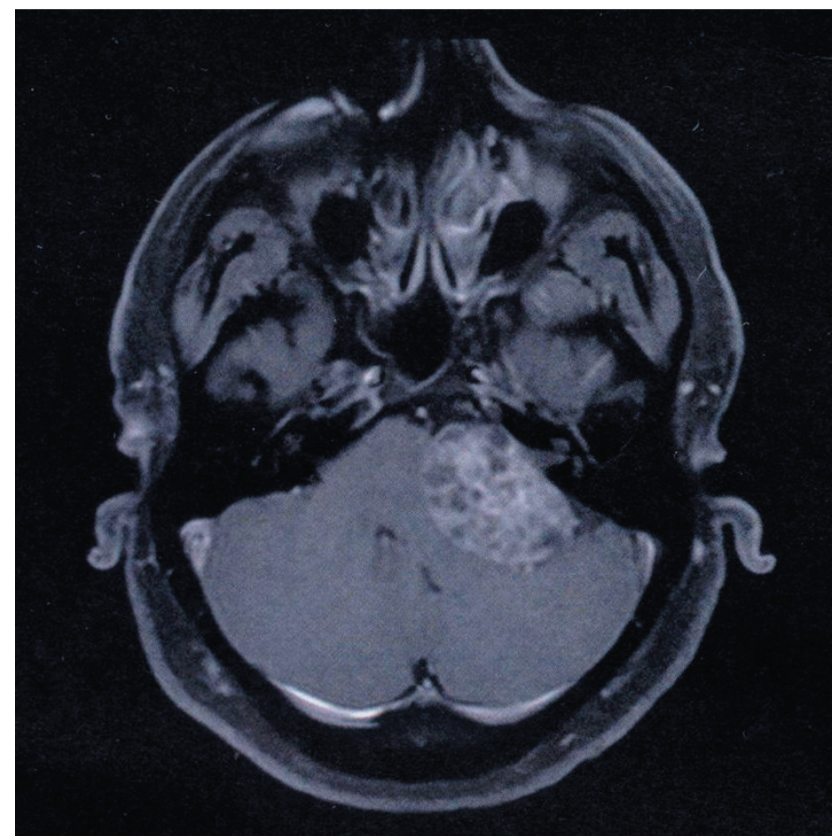

Figura 1. RM (T1 con gadolinio) pre-operatoria que muestra una voluminosa masa en el ángulo ponto-cerebeloso izquierdo que provoca marcado desvio y rotación del tronco cerebral.

derecha. (Fig 1). La hipótesis diagnóstica más probable fue la de neurinoma del acústico.

El paciente fue operado a través de un acceso retrosigmoideo, en posición de semi-sentado. El tumor fue resecado en su totalidad (Fig. 2), no siendo posible preservar los nervios facial y acústico. El examen anatomopatológico confirmó el diagnóstico preoperatorio de schwannoma.

Tras un postoperatorio sin complicaciones, se inició una retirada progresiva de la medicación. La cuarta semana después de la exéresis del tumor fue realizada una anastomosis hipogloso-facial izquierda. El dolor facial y la hipoestesia remitieron completamente 3 meses después de la cirugía. 2 años después el paciente se mantiene sin dolor facial, no está medicado y presenta una parálisis facial grado III de Brudny y una pérdida total de la audición a la izquierda.

\section{Discusión}

A pesar de la uniformidad en su presentación clínica, el mecanismo subyacente a la neuralgia del trigémino permanece poco claro y ha sido asociado a múltiples agentes etiológicos y mecanismos fisiopatológicos.

Fue Dandy ${ }^{2}$ el primer autor que desarrolló la teoría de la compresión vascular de la raíz como mecanismo causal de la neuralgia idiopática. Posteriormente Jannetta ${ }^{8}$ popularizó esta teoría gracias a la generalización del uso de microscopio quirúrgico. Para este autor, la causa de la

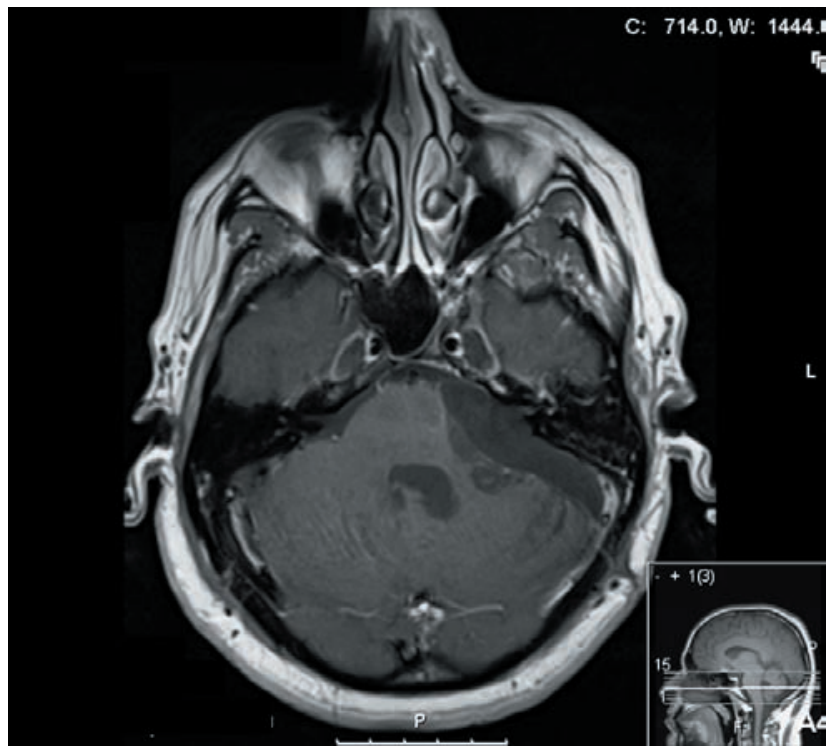

Figura 2. RM (T1 con gadolinio) realizada 6 meses después de la cirugía. Sin residuo tumoral ni desplazamiento del tronco cerebral.

neuralgia del trigémino es una compresión o deformación de la raíz dorsal del nervio trigémino en su zona de entrada en la protuberancia ocasionado por bucles vasculares.

En el año 1910, Weisenburg ${ }^{12}$ describió por primera vez la relación entre neuralgia del trigémino y tumores ipsilaterales del ángulo ponto-cerebeloso. Varios estudios clínicos han señalado que, en torno al $1 \%$ de los pacientes con neuralgia del trigémino presentan un tumor del ángulo ponto-cerebeloso del mismo lado. Sin embargo, la neuralgia del trigémino provocada por un tumor del ángulo ponto-cerebeloso contralateral es muy infrecuente, existiendo varias teorías que explicarían su mecanismo etiopatogénico. Para algunos autores ${ }^{9}$ la principal causa del dolor es la compresión contralateral de la raíz trigeminal contra la duramadre provocada por el desplazamiento lateral del tronco del encéfalo. Otros autores ${ }^{6}$ defienden que, más importante que el desplazamiento lateral del tronco, lo es el grado de rotación que la masa contralateral provoca en el tronco, y el consecuente desplazamiento lateral de la arteria basilar que a su vez somete a tensión mecánica a las ramas vasculares dependientes de ésta, pudiendo permitir que un bucle vascular entre en contacto con la raíz desencadenándose así la neuralgia. Esta teoría podría explicar cómo algunas lesiones de menor tamaño, pero situadas de tal manera que impriman cierto desplazamiento rotacional, pueden generar una neuralgia contralateral.

En el caso que nos compromete es posible que ambos mecanismos participen en la etiología del dolor puesto que los autores observaron tanto compresión lateral como rotación troncoencefálica. 
C. Chamadoira y col

Por otra parte no hay que desestimar que la neuralgia trigeminal no sea secundaria a la afectación de la raíz sino provocada por la lesión compresiva del propio núcleo trigeminal que como sabemos presenta una amplia extensión en el tronco, aceptando así una hipótesis del dolor que implicaría mecanismos centrales y no de afectación periférica ${ }^{11}$.

Es posible considerar que existan otros mecanismos desencadenantes del dolor contralateral, ya que en algunos casos, como los descritos por Grigoryan y col. ${ }^{5}$, existe una persistencia del dolor después de la escisión del tumor. No obstante, nuestra experiencia coincide con la de Findler ${ }^{3}$ y Cheng ${ }^{1}$, en que el dolor facial desaparece después de la extracción de la lesión. De este modo, la mejor estrategia para el tratamiento de este tipo de neuralgia es la escisión total del tumor contralateral ${ }^{4,7,10}$ y esperar por la desaparición de los síntomas. No obstante, la extirpación parcial de la lesión con reducción volumétrica de la misma (seguida o no de terapias complementarias) puede significar un alivio a largo plazo de la neuralgia y puede contemplarse como una alternativa en aquellos pacientes en los que no es posible un tratamiento radical o en los que el mismo puede significar un deterioro funcional relevante.

\section{Conclusiones}

Los tumores de la fosa posterior pueden, de forma excepcional, presentarse en forma de neuralgia del trigémino contralateral confundiendo su diagnóstico. En estos casos debe ser abordado inicialmente el tumor y no la neuralgia. La escisión total de la lesión suele curar el dolor contralateral. En los casos de recurrencia de la neuralgia debe ser considerada la descompresión neurovascular del nervio trigémino.

\section{Bibliografía}

1. Cheng, W.C., Chang, C.N.: Trigeminal neuralgia caused by contralateral supratentorial meningioma. J Clin Neurosci 2008; 15: 1162-1163.

2. Dandy, W.E.: Concerning the cause of trigeminal neuralgia. Am J Surg 1934; 24: 447-455.

3. Findler, G., Feinsod, M.: Reversible facial pain due to
Neurocirugía 2010; $21: 50-52$

hydrocephalus with trigeminal somatosensory evoked response changes. Case report. J Neurosurg 1982; 57: 267-269.

4. Florensa, R., Llovet, J., Pou, A., Galitó, E., Vilato, J., Colet, S.: Contralateral trigeminal neuralgia as a false localizing sign in intracranial tumours. Neurosurgery 1987; 20: 1-3.

5. Grigoryan, Y.A., Onopchenko, C.V.: Persistent trigeminal neuralgia after removal of contralateral posterior cranial fossa tumor. Report of two cases. Surg Neurol 1999; 52: 55-60.

6. Haddad, F.S., Taha, J.M.: An unusual cause for trigeminal neuralgia: contralateral meningioma of the posterior fossa. Neurosurgery 1990; 26: 1033-1038.

7. Hamby, W.B.: Trigeminal neuralgia due to contralateral tumors of the posterior cranial fossa. Report of 2 cases. J Neurosurg 1947; 4: 179-182.

8. Jannetta, P.J.: Arterial compression of the trigeminal nerve at the pons in patients with trigeminal neuralgia. $\mathrm{J}$ Neurosurg 1967; 26: 159-162.

9. Paillas, J.E., Pellet, W., Janny, P., Tournilhac, M., Komminoth, J.: L'atteinte contre-latérale des nerfs craniens au cours des tumeurs de la fosse postérieure. Rev. Neurologique 1969;121: 452-464.

10. Snow, R.B., Fraser, R.A.: Cerebellopontine angle tumour causing contralateral trigeminal neuralgia: A case report. Neurosurgery 1987; 21: 84-86.

11. Tsai, B.M.D., Wang, A.D.J.: Trigeminal neuralgia caused by a contralateral acoustic neuroma: a case report. Chang Gung Med J 1988; 22: 281-284.

12. Weisenburg, T.H.: Cerebello-pontine tumor diagnosed for six years as tic douloureux. The symptoms of irritation of the ninth and twelfh cranial nerve. JAMA 1910; 54: 16001604.

13. Wepsic, T.G.: Tic douloureux: etiology, refined treatment. N Engl J Med 1973; 288:680-681.

Chamadoira, C,; Cerejo, A.; Duarte, F.; Vaz, R.: Neuralgia del trigémino provocada por un tumor del ángulo pontocerebeloso contralateral. Caso clínico. Neurocirugía 2010; 21: 50-52.

Correspondencia: Clara Chamadoira Villaverde, Neurosurgery Department, Hospital de São João, Alameda Professor Hernâni Monteiro, 4200-319, Porto, Portugal.

E-mail: clarachamadoira@gmail.com 